\title{
Multiscale Modeling and Computation of Optically Manipulated Nano Devices
}

\author{
Gang $\mathrm{BaO}^{\mathrm{a}}$, Di Liu ${ }^{\mathrm{b}}$, Songting Luo ${ }^{\mathrm{c}, *}$ \\ ${ }^{a}$ Department of Mathematics, Zhejiang University, Hangzhou 310027, China \\ ${ }^{b}$ Department of Mathematics, Michigan State University, East Lansing, MI 48824, USA. \\ ${ }^{c}$ Department of Mathematics, Iowa State University, Ames, IA 50011, USA.
}

\begin{abstract}
We present a multiscale modeling and computational scheme for opticalmechanical responses of nanostructures. The multi-physical nature of the problem is a result of the interaction between the electromagnetic (EM) field, the molecular motion, and the electronic excitation. To balance accuracy and complexity, we adopt the semi-classical approach that the EM field is described classically by the Maxwell equations, and the charged particles follow the Schröidnger equations quantum mechanically. To overcome the numerical challenge of solving the high dimensional multi-component manybody Schrödinger equations, we further simplify the model with the Ehrenfest molecular dynamics to determine the motion of the nuclei, and use the TimeDependent Current Density Functional Theory (TD-CDFT) to calculate the excitation of the electrons. This leads to a system of coupled equations that computes the electromagnetic field, the nuclear positions, and the electronic current and charge densities simultaneously. In the regime of linear responses, the resonant frequencies initiating the out-of-equilibrium optical-mechanical responses can be formulated as an eigenvalue problem. A self-consistent multiscale method is designed to deal with the well separated space scales. The isomerization of Azobenzene is presented as a numerical example.
\end{abstract}

\footnotetext{
*Corresponding author

Email addresses: baog@zju.edu.cn (Gang Bao), richardl@math.msu.edu (Di Liu), luos@iastate.edu (Songting Luo)

${ }^{1}$ Research supported by NSF-DMS 0968360, NSF-DMS 1211292, ONR-N00014-12-10319, NSFC-91130004, and a special research grant from Zhejiang University.

${ }^{2}$ Research supported by NSF-DMS 1418959 and NSF-DMS 0968360.

${ }^{3}$ Research supported by NSF-DMS 1418908.
} 
Keywords: Optical responses, Nanostructures, Multiscale modeling and computation, Maxwell equations, Ehrenfest dynamics, Time-dependent current density functional theory, Resonant condition, Eigenvalue problem

\section{Introduction}

Optically manipulated nano devices have attracted a lot of recent interest with applications in solar energy harvesting, molecular engineering, molecular sensing and non-invasive regulation of intra-cellular reactions $[12,16$, 30, 29]. One such example is a photoresponsive DNA nanomotor enhanced by silver nanowires as illustrated in [30]. Illuminated by ultraviolet (UV) and visible lights, the nanomotor can switch back and forth between 'open' and 'loop' states, thereby converting photonic energy to mechanical energy. The mechanism is facilitated by incorporating azobenzene moieties that can change conformational structure through the cis-trans isomerization. The efficiency of the conversion can be significantly enhanced by a plasmonic near-field coupling with silver nanoparticles, due to the spectral overlap between the azobenzene absorption band and plasmonic resonances of silver nanowires. Recent applications include photon regulated enzymatic nanostructures, drug releasing from nanocontainers, mRNA detection in living cells, etc.. A quantitative investigation of this type of nano scale opticalmechanical systems will obviously help to find optimal designing and novel applications.

From the modeling point of view, when the system is of nano scale, the macroscopic theory for the electromagnetic (EM) field fails to capture the microscopic and nonlocal character of the light-matter interactions, which suggests the quantum mechanical description of the current and charge densities. Quantum Electrodynamics (QED) [7] gives a complete description of the interactions between the EM field and the charged particles, but has limited applications to complex systems due to its high computational expenses. Semi-classical theories $[23,17,6]$ combine the classical treatment of the EM field and the first principle approach to the charged particles. Different from QED, in a semi-classical theory, the EM field is not quantized, therefore lower computational cost is required. The EM field as well as the current and charge densities are determined self-consistently through coupled Maxwell and Schrödinger equations. However semi-classical theories are still prohibitively expensive for most applications of interest in nano op- 
tics since the high dimensional many-body Schrödinger equations still need to be numerically solved.

In a previous paper [2], we incorporated Time-Dependent Current Density Functional Theory (TD-CDFT) $[14,22,11]$ into the framework of the semi-classical optical response theory, thereby avoiding the computational cost of solving the Schrödinger equation. In the Density Functional Theory (DFT) for the ground state of electrons, a one-to-one correspondence up to an arbitrary constant between the external potential and the ground state electron density has been established in the seminal work of Hohenberg and Kohn [14]. Hence, the wavefunction can be obtained as a functional of the electron density, which allows the evaluation of all observables of the system. Similar results have been extended to the case of time evolutionary electronic structures in the form of Time-Dependent Density Functional Theory (TDDFT) by Runge and Gross [22], and later to the situation of external electric and magnetic fields with arbitrary time dependence by Ghosh and Dhara [11] in the form of TD-CDFT, where the current density is introduced as the fundamental variable. A practical Kohn-Sham (KS) system [18], which is a numerically tractable single-particle system under effective external potentials, was introduced to compute the electron and current densities such that they are the same as those in the original interacting system under consideration.

We call the system obtained in [2] the Density Functional semi-classical theory for nano-optics, which is a combination of the semi-classical theory with TD-CDFT and can be formulated as coupled Maxwell-Kohn-Sham (Maxwell-KS) equations (also see [3, 19, 8]). In the regime of linear responses, a system of linear equations for concurrently determining the EM field, the current density and the electron density can be derived. Moreover, the zero eigenvalue problem of the linear system corresponds to the resonant eigenmodes [6] of the nano-optical response. To deal with the disparate space scales of the system, we proposed a multiscale scheme which can solve the system self-consistently by allowing communications between the macrosolver for Maxwell equations and the micro-solver for Kohn-Sham equations. Numerical experiments with simple molecular structures such as Methane have shown validity of the model as well as efficiency and accuracy of the scheme.

In this work, we study the light driven nano devices by further introducing the molecular motion into the system through the Ehrenfest molecular dynamics [21], where the excitation of the electrons and the motion of the 
nuclei are separated under the single-determinant approximation and molecular dynamics is obtained by the mean-field approximation [24]. The coupled system of classical electromagnetism and Ehrenfest molecular dynamics along with TD-CDFT, which we refer as the Maxwell-Ehrenfest-Kohn-Sham equations, provides a computationally practical model to investigate the optically manipulated nanostructures. Within the linear response regime, we formulate a linear system that determines the induced EM field, nuclear positions, and current and charge densities simultaneously. Similar to [2], we can determine the resonant conditions initiating the molecular motion by solving an eigenvalue problem. The multiscale scheme introduced in [2] will also be generalized to deal with the three level system with well-separated electromagnetic, molecular and electronic scales.

The rest of the paper is organized as follows. In Section 2, we first incorporate Ehrenfest molecular dynamics into the Density Functional semi-classical Theory. Then in Section 3, we present the linear response formulation for the coupled Maxwell-Ehrenfest-Kohn-Sham system. The multiscale solver for the linear system is described in detail in Section 4. Finally in Section 5, Azobenzene isomerization [13] will be presented as numerical examples. Throughout the paper, we will represent vector variables in boldface notations and scalar variables in the normal font.

\section{The Maxwell-Ehrenfest-Kohn-Sham Model}

We first briefly review the semi-classical theory for nano scale light-matter interactions, the Ehrenfest dynamics and TD-CDFT separately, then formulate the Maxwell-Ehrenfest-Kohn-Sham equation by coupling these theories.

\subsection{Semi-classical theory}

In the semi-classical theory [6], the evolution of the EM field is determined by Maxwell equations in terms of the vector and scalar potentials $\{\mathbf{A}, \phi\}$ under the Coulomb gauge $\nabla \cdot \mathbf{A}=0$ with the following form:

$$
\begin{aligned}
\frac{1}{c^{2}} \frac{\partial^{2} \mathbf{A}(\mathbf{r}, t)}{\partial t^{2}}-\nabla^{2} \mathbf{A}(\mathbf{r}, t)+\frac{1}{c} \frac{\partial(\nabla \phi(\mathbf{r}, t))}{\partial t} & =\frac{4 \pi}{c} \mathbf{j}(\mathbf{r}, t), \\
-\nabla^{2} \phi(\mathbf{r}, t) & =4 \pi \rho(\mathbf{r}, t),
\end{aligned}
$$


where $c$ is the speed of light in vacuum, and $\mathbf{j}$ and $\rho$ are the current and charge densities related by the continuity equation

$$
\nabla \cdot \mathbf{j}(\mathbf{r}, t)+\frac{\partial \rho(\mathbf{r}, t)}{\partial t}=0
$$

The electric and magnetic fields $\mathbf{E}$ and $\mathbf{B}$ can be evaluated by

$$
\mathbf{E}=-\nabla \phi-\frac{1}{c} \frac{\partial \mathbf{A}}{\partial t}, \quad \mathbf{B}=\nabla \times \mathbf{A}
$$

Quantum mechanically, the motion of a system of $N$ charged particles is governed by the time-dependent Schrödinger equation [7]

$$
\iota \hbar \frac{\partial \Psi\left(\mathbf{r}_{1}, \ldots, \mathbf{r}_{N}, t\right)}{\partial t}=H_{M} \Psi\left(\mathbf{r}_{1}, \ldots, \mathbf{r}_{N}, t\right)
$$

where $\iota=\sqrt{-1}, \hbar$ is the reduced Planck constant, and the general nonrelativistic Hamiltonian takes the form

$$
H_{M}\left(\left\{\mathbf{r}_{l}\right\},\left\{\mathbf{p}_{l}\right\}, t\right)=\sum_{l=1}^{N} \frac{1}{2 m_{l}}\left[\mathbf{p}_{l}-\frac{e_{l}}{c} \mathbf{A}\left(\mathbf{r}_{l}\right)\right]^{2}+\frac{1}{2} \sum_{l \neq l^{\prime}} \frac{e_{l} e_{l^{\prime}}}{\left|\mathbf{r}_{l}-\mathbf{r}_{l^{\prime}}\right|}
$$

with $e_{l}, m_{l}, \mathbf{r}_{l}$ and $\mathbf{p}_{l}$ being the charge, mass, coordinate and conjugate momentum of the $l^{\text {th }}$ particle, respectively. The initial state before the incident light is applied, denoted as $\Psi_{0}$, is assumed to satisfy

$$
H_{0} \Psi_{0}\left(\mathbf{r}_{1}, \ldots, \mathbf{r}_{N}\right)=E_{0} \Psi_{0}\left(\mathbf{r}_{1}, \ldots, \mathbf{r}_{N}\right),
$$

with ground state energy $E_{0}$ and

$$
H_{0}\left(\left\{\mathbf{r}_{l}\right\},\left\{\mathbf{p}_{l}\right\}\right)=\sum_{l=1}^{N} \frac{1}{2 m_{l}} \mathbf{p}_{l}^{2}+\frac{1}{2} \sum_{l \neq l^{\prime}} \frac{e_{l} e_{l^{\prime}}}{\left|\mathbf{r}_{l}-\mathbf{r}_{l^{\prime}}\right|}
$$

The current and charge densities, $\{\mathbf{j}(\mathbf{r}, t), \rho(\mathbf{r}, t)\}$, can be computed as expectations with respect to the solution of the Schrödinger equation, i.e.,

$$
\mathbf{j}(\mathbf{r}, t)=\langle\Psi|\hat{\mathbf{j}}| \Psi\rangle \quad \text { and } \quad \rho(\mathbf{r}, t)=\langle\Psi|\hat{\rho}| \Psi\rangle
$$


with the current and charge density operators $\{\hat{\mathbf{j}}, \hat{\rho}\}$ given by

$$
\begin{aligned}
& \hat{\mathbf{j}}=\sum_{l=1}^{N} \frac{e_{l}}{2 m_{l}}\left[\mathbf{p}_{l} \mathrm{I}\left(\mathbf{r}-\mathbf{r}_{l}\right)+\mathrm{I}\left(\mathbf{r}-\mathbf{r}_{l}\right) \mathbf{p}_{l}\right]-\sum_{l=1}^{N} \frac{e_{l}^{2}}{m_{l} c} \mathbf{A}\left(\mathbf{r}_{l}, t\right), \\
& \hat{\rho}=\sum_{l=1}^{N} e_{l} \mathrm{I}\left(\mathbf{r}-\mathbf{r}_{l}\right),
\end{aligned}
$$

where $\mathrm{I}(\cdot)$ is the Dirac delta function.

In the semi-classical model, the light-matter interaction is completely described by $(\mathbf{A}, \phi)$ and $\Psi$ as a coupled system of Maxwell equations (1) and Schrödinger equation (4), through the current and charge densities by (7). Therefore the system must be solved self-consistently to accurately describe the interactions.

\subsection{Ehrenfest molecular dynamics}

Let $\overline{\mathbf{r}}=\left(\mathbf{r}_{1}, \ldots, \mathbf{r}_{N_{e}}\right)$ and $\overline{\mathbf{R}}=\left(\mathbf{R}_{1}, \ldots, \mathbf{R}_{N_{n}}\right)$ denote the positions of the electrons and nuclei with $N_{e}$ and $N_{n}$ being the total number of electrons and nuclei respectively. The electronic and nuclear wavefunctions are given by $\psi$ and $\Phi$ respectively, and $e, m, \mathbf{r}_{l}, \mathbf{p}_{\mathbf{r}_{l}}$ (resp. $Z_{k}, M_{k}, \mathbf{R}_{k}, \mathbf{p}_{\mathbf{R}_{k}}$ ) represent the charge, mass, coordinate, and conjugate momentum of coordinate of $l^{\text {th }}$ electron (resp. $k^{t h}$ nucleus). The Coulomb interactions can be partitioned into

$$
\begin{aligned}
W_{e e}(\overline{\mathbf{r}})= & \frac{1}{2} \sum_{j \neq k}^{N_{e}} \frac{e^{2}}{\left|\mathbf{r}_{j}-\mathbf{r}_{k}\right|}, \quad W_{n n}(\overline{\mathbf{R}})=\frac{1}{2} \sum_{j \neq k}^{N_{n}} \frac{Z_{j} Z_{k}}{\left|\mathbf{R}_{j}-\mathbf{R}_{k}\right|}, \\
& W_{e n}(\overline{\mathbf{r}}, \overline{\mathbf{R}})=\sum_{j=1}^{N_{e}} \sum_{k=1}^{N_{n}} \frac{e Z_{k}}{\left|\mathbf{r}_{j}-\mathbf{R}_{k}\right|} .
\end{aligned}
$$

In $[21,24]$, it is shown that one can separate the nuclear and electronic degrees of freedom with the following product ansatz for the solution of (4):

$$
\Psi(\overline{\mathbf{r}}, \overline{\mathbf{R}}, t)=\psi(\overline{\mathbf{r}}, t) \Phi(\overline{\mathbf{R}}, t) \exp \left[\frac{\iota}{\hbar} \int_{t_{0}}^{t}\left\langle H_{e}\right\rangle_{\overline{\mathbf{r}}, \overline{\mathbf{R}}}\right]
$$


where $\langle\cdot\rangle_{z}$ denotes expectation values with respect to $z$ variable and

$$
H_{e}=\sum_{l=1}^{N_{e}} \frac{1}{2 m}\left(\mathbf{p}_{\mathbf{r}_{l}}-\frac{e}{c} \mathbf{A}\left(\mathbf{r}_{l}\right)\right)^{2}+W_{e e}+W_{n n}+W_{e n} .
$$

Then the motion of electrons is determined by the electronic Schrödinger equation

$$
\begin{aligned}
& \iota \hbar \frac{\partial \psi(\overline{\mathbf{r}}, t)}{\partial t} \\
& \quad=\left(\sum_{l=1}^{N_{e}} \frac{1}{2 m}\left(\mathbf{p}_{\mathbf{r}_{l}}-\frac{e}{c} \mathbf{A}\left(\mathbf{r}_{l}\right)\right)^{2}+\left\langle W_{e e}+W_{n n}+W_{e n}\right\rangle_{\overline{\mathbf{R}}}\right) \psi(\overline{\mathbf{r}}, t),
\end{aligned}
$$

and the motion of nuclei is determined by the nuclear Schrödinger equation

$$
\iota \hbar \frac{\partial \Phi(\overline{\mathbf{R}}, t)}{\partial t}=\left(\sum_{k=1}^{N_{n}} \frac{1}{2 M_{k}}\left(\mathbf{p}_{\mathbf{R}_{k}}-\frac{Z_{k}}{c} \mathbf{A}\left(\mathbf{R}_{k}\right)\right)^{2}+\left\langle H_{e}\right\rangle_{\overline{\mathbf{r}}}\right) \Phi(\overline{\mathbf{R}}, t) .
$$

Equations (12) and (13) are also known as the Time-Dependent Self-Consistent Field (TD-SCF) equations.

The motion of nuclei is further approximated with the mean-field approximation. Assume that the nuclear wavefunction has the WKB ansatz $[21]$

$$
\Phi(\overline{\mathbf{R}}, t)=A(\overline{\mathbf{R}}, t) e^{\iota S(\overline{\mathbf{R}}, t) / \hbar}+O(\hbar), \quad \text { as } \hbar \rightarrow 0,
$$

where $A$ is the amplitude and $S$ is the phase. The approximation (14) is valid locally in time, and a time obstruction for the validity is $\approx \log \hbar^{-1}$ that is called the "Ehrenfest time". It is easy to derive that $S$ satisfies HamiltonJacobi equation such that

$$
\begin{aligned}
\frac{\partial S(\overline{\mathbf{R}}, t)}{\partial t} & +\sum_{k=1}^{N_{n}} \frac{1}{2 M_{k}}\left|\nabla_{\mathbf{R}_{k}} S(\overline{\mathbf{R}}, t)\right|^{2} \\
& +\sum_{k=1}^{N_{n}} \frac{1}{2 M_{k}}\left(-\frac{2 Z_{k}}{c} \mathbf{A}\left(\mathbf{R}_{k}, t\right) \cdot \nabla_{\mathbf{R}_{k}} S(\overline{\mathbf{R}}, t)+\frac{Z_{k}^{2}}{c^{2}} \mathbf{A}^{2}\left(\mathbf{R}_{k}, t\right)\right) \\
& +\left\langle H_{e}\right\rangle_{\overline{\mathbf{r}}}=0 .
\end{aligned}
$$


Let $\mathbf{P}_{k}=\nabla_{\mathbf{R}_{k}} S(\overline{\mathbf{R}}, t)$, the Hamiltonian dynamics for solving (15) has the following form:

$$
\left\{\begin{aligned}
\frac{d \mathbf{R}_{k}}{d t}= & \frac{\mathbf{P}_{k}}{M_{k}}-\frac{Z_{k}}{M_{k} c} \mathbf{A}\left(\mathbf{R}_{k}, t\right) \\
\frac{d \mathbf{P}_{k}}{d t}= & \frac{Z_{k}}{M_{k} c} \nabla_{\mathbf{R}_{k}} \mathbf{A}\left(\mathbf{R}_{k}, t\right) \cdot \mathbf{P}_{k}-\frac{Z_{k}^{2}}{M_{k} c^{2}} \mathbf{A}\left(\mathbf{R}_{k}, t\right) \cdot \nabla_{\mathbf{R}_{k}} \mathbf{A}\left(\mathbf{R}_{k}, t\right) \\
& -\nabla_{\mathbf{R}_{k}}\left\langle H_{e}\right\rangle_{\overline{\mathbf{r}}}
\end{aligned}\right.
$$

from which we can derive

$$
\begin{aligned}
M_{k} \frac{d^{2} \mathbf{R}_{k}}{d t^{2}}= & \frac{-Z_{k}}{c}\left(\frac{\partial \mathbf{A}\left(\mathbf{R}_{k}, t\right)}{\partial t}\right) \\
& -\nabla_{\mathbf{R}_{k}}\left(W_{n n}+\int \rho_{e}(\mathbf{r}, t) W_{e n}(\mathbf{r}, \overline{\mathbf{R}}) d \mathbf{r}\right)
\end{aligned}
$$

for $k=1, \ldots, N_{n}$, and

$$
W_{e n}(\mathbf{r}, \overline{\mathbf{R}})=\sum_{k=1}^{N_{n}} \frac{e Z_{k}}{\left|\mathbf{r}-\mathbf{R}_{k}\right|}
$$

The coupled equation (12) and (17) is referred as Ehrenfest dynamics of electrons and nuclei.

\subsection{Time-dependent current density functional theory}

For computing the charge and current densities due to electronic motion, denoted as $\left\{\rho_{e}, \mathbf{j}_{e}\right\}$, instead of solving the many-body Schrödinger equation (12), TD-CDFT is adopted for approximations. The advantage of TD-CDFT is that by restricting to the charge and current densities that are functions of only three-dimensional (3-D) spatial variables, the computational cost can be greatly reduced.

DFT was originally designed to calculate the ground state electron density for a system satisfying time-independent Schrödinger equation (6). Following the seminal work of Hohenberg and Kohn [14] and Kohn and Sham [18], the Kohn-Sham (KS) system is given as

$$
H_{0}^{K S} \psi_{l}(\mathbf{r})=\epsilon_{l} \psi_{l}(\mathbf{r}), \quad \text { for } l=1, \ldots, N_{e},
$$


with

$$
H_{0}^{K S}(\mathbf{r}, \mathbf{p})=\frac{1}{2 m} \mathbf{p}^{2}+v_{K S}(\mathbf{r}) .
$$

The effective KS potential $v_{K S}(\mathbf{r})$ is given by

$$
v_{K S}(\mathbf{r})=v(\mathbf{r})+v_{H}(\mathbf{r})+v_{x c}(\mathbf{r})
$$

where $v(\mathbf{r})$ is the external potential due to nuclear attraction, and $v_{H}(\mathbf{r})$ is the Hartree potential given as

$$
v_{H}(\mathbf{r})=e \int \frac{\rho_{e}\left(\mathbf{r}^{\prime}\right)}{\left|\mathbf{r}-\mathbf{r}^{\prime}\right|} d \mathbf{r}^{\prime}
$$

$v_{x c}(\mathbf{r})$ represents the exchange-correlation (xc) potential containing the manybody effects and is a functional of $\rho_{e}$. Once the KS system (19) is solved, which gives the ground state orbitals $\left\{\psi_{l}(\mathbf{r})\right\}$ with energy $\left\{\epsilon_{l}\right\}$, the charge density $\rho_{e}$ can be computed by

$$
\rho_{e}(\mathbf{r})=\sum_{l=1}^{N_{e}} e f_{l}\left|\psi_{l}(\mathbf{r})\right|^{2},
$$

with $f_{l}$ being the occupation number of orbital $\psi_{l}$.

DFT has been extended as TD-DFT by Runge and Gross [22] for timedependent systems and as TD-CDFT by Ghosh and Dhara [11] for timedependent systems under the influence of both electric and magnetic fields. For a system satisfying the Schrödinger equation (12), a system of timedependent KS equations can also be constructed in TD-CDFT [11] in the following form:

$$
\iota \hbar \frac{\partial \psi_{l}(\mathbf{r}, t)}{\partial t}=H_{M}^{K S} \psi_{l}(\mathbf{r}, t), \quad \text { for } l=1, \ldots, N_{e}
$$

with the Hamiltonian

$$
H_{M}^{K S}(\mathbf{r}, \mathbf{p}, t)=\frac{1}{2 m}\left[\mathbf{p}-\frac{e}{c} \mathbf{A}_{K S}(\mathbf{r}, t)\right]^{2}+v_{K S}(\mathbf{r}, t) .
$$

The time-dependent KS potentials are given by

$$
v_{K S}(\mathbf{r}, t)=v(\mathbf{r}, t)+v_{H}(\mathbf{r}, t)+v_{x c}(\mathbf{r}, t),
$$


where $v(\mathbf{r}, t), v_{x c}(\mathbf{r}, t)$ and $v_{H}(\mathbf{r}, t)$ represent respectively the time-dependent external potential, scalar xc-potential and Hartree potential analogous to those in DFT,

$$
\mathbf{A}_{K S}(\mathbf{r}, t)=\mathbf{A}(\mathbf{r}, t)+\mathbf{A}_{x c}(\mathbf{r}, t)
$$

with $\mathbf{A}$ being the vector potential, and $\mathbf{A}_{x c}(\mathbf{r}, t)$ is the vector xc-potential that is a functional of the current density. Once the KS system (24) is solved, we can compute the charge density $\rho_{e}(\mathbf{r}, t)$ by $(23)$ with $\left\{\psi_{l}(\mathbf{r}, t)\right\}$, and the current density by

$$
\begin{aligned}
\mathbf{j}_{e}(\mathbf{r}, t)=-\frac{\iota \hbar e}{2 m} & \sum_{l=1}^{N_{e}} f_{l}\left[\psi_{l}^{*}(\mathbf{r}, t) \nabla \psi_{l}(\mathbf{r}, t)-\psi_{l}(\mathbf{r}, t) \nabla \psi_{l}^{*}(\mathbf{r}, t)\right] \\
& -\frac{e}{m c} \rho_{e}(\mathbf{r}, t) \mathbf{A}_{K S}(\mathbf{r}, t) .
\end{aligned}
$$

In practice, both the scalar xc-potential $v_{x c}$ and the vector xc-potential $\mathbf{A}_{x c}$ need to be approximated. For applications here, the local density approximation (LDA) and the adiabatic local density approximation (ALDA) are used for $v_{x c}$ in the ground state case and the time-dependent case, respectively [10, 20], with parametrization of Vosko, Wilk, and Nusair (VWN) [28]. For the vector xc-potential $\mathbf{A}_{x c}$, the Vignale-Kohn (VK) functional $[25,26]$ is used.

\subsection{The coupled Maxwell-Ehrenfest-Kohn-Sham system}

The coupled system that is capable of describing the interactions between the induced EM field, the molecular motion and the electronic excitation consists of Maxwell equations (1) for the EM field, the Ehrenfest dynamics for the nuclei (17), and time-dependent KS system for the electrons (24). We refer to this system as Maxwell-Ehrenfest-Kohn-Sham equations which must be solved self-consistently. In (1), the current and charge densities are partitioned into the electronic and nuclear contributions such as

$$
\mathbf{j}(\mathbf{r}, t)=\mathbf{j}_{e}(\mathbf{r}, t)+\mathbf{j}_{n}(\mathbf{r}, t), \rho(\mathbf{r}, t)=\rho_{e}(\mathbf{r}, t)+\rho_{n}(\mathbf{r}, t) .
$$

While the electronic current and charge densities can be obtained by the KS equation (24), the current and charge densities due to nuclear motion, 
denoted as $\left(\mathbf{j}_{n}, \rho_{n}\right)$, are given as

$$
\rho_{n}(\mathbf{R}, t)=\sum_{k=1}^{N_{n}} Z_{k} \mathrm{I}\left(\mathbf{R}-\mathbf{R}_{k}\right), \quad \mathbf{j}_{n}(\mathbf{R}, t)=\sum_{k=1}^{N_{n}} Z_{k} \mathbf{v}_{k} \mathrm{I}\left(\mathbf{R}-\mathbf{R}_{k}\right),
$$

with $\mathbf{v}_{k}=\frac{d \mathbf{R}_{k}}{d t}$. The so-obtained Maxwell-Ehrenfest-Kohn-Sham system is multi-physical in nature, as a result of the coupling of physical processes at different time and space scales. It also poses a numerical challenge due to well separated time and space scales of these processes.

The Ehrenfest molecular dynamics treats the electronic dynamics quantum mechanically and the nuclear dynamics classically with the nuclear quantum states reduced to classical trajectories governed by Hamilton's equations. This approximation works well for situations where a single path of the nuclear dynamics is dominant, for ultrafast processes before any significant amount of level crossing can take place, and for situations when a large number of similar electronic excitations are involved and the nuclear dynamics is governed by an averaged force [24].

\section{Linear Response Theory of the Maxwell-Ehrenfest-Kohn-Sham System}

To investigate the resonance effects of light on the molecular structure, we consider the linear response regime by treating the incident light as resonant perturbations driving the system away from the local minimum of the molecular energy landscape. To this end, we assume the nuclei are at equilibrium position $\overline{\mathbf{R}}_{0}=\left(\mathbf{R}_{01}, \ldots, \mathbf{R}_{0 N_{n}}\right)$ before the incident light is switched on. In particular, tackling the system within the linear response regime allows us to solve the coupled system in the frequency domain $(\omega)$ after the Fourier transform given for any function $\mathbf{f}$ by

$$
\mathbf{f}(t)=\int \mathbf{f}(\omega) e^{-\imath \omega t} d \omega
$$

For consistency, incident and ground state quantities are indicated by adding subscript $(\cdot)_{0}$, e.g., $\mathbf{A}_{0}$ represents the incident light, $\left(\rho_{e}\right)_{0}$ is the ground state electron density and $\left(W_{n n}\right)_{0}=W_{n n}\left(\overline{\mathbf{R}}_{0}\right)$. Induced responses will be denoted with prefix $\delta$, i.e., $\delta \mathbf{A}$ for induced light. For simplicity, atomic units (a.u., $-e=\hbar=m=1$ ) are adopted in this and following sections. 
Assuming the nuclei to be in the equilibrium state before the applied EM field, with the first order approximation that $\mathbf{R}_{k}=\mathbf{R}_{0 k}+\delta \mathbf{R}_{k}$ and $\mathbf{A}=\mathbf{A}_{0}+\delta \mathbf{A}$, the linearized Hamilton's equation (17) takes the following form:

$$
\begin{aligned}
-\omega^{2} M_{k} \delta \mathbf{R}_{k}=Z_{k} & \frac{\iota \omega}{c}\left(\mathbf{A}_{0}+\nabla_{\mathbf{R}_{k}} \mathbf{A}_{0} \delta \mathbf{R}_{k}+\delta \mathbf{A}\right) \\
& -\sum_{j=1}^{N_{n}} \nabla_{\mathbf{R}_{k}} \nabla_{\mathbf{R}_{j}}\left(W_{n n}\right)_{0} \delta \mathbf{R}_{j} \\
& -\int d \mathbf{r}\left(\rho_{e}\right)_{0}(\mathbf{r}) \sum_{j=1}^{N_{n}} \nabla_{\mathbf{R}_{k}} \nabla_{\mathbf{R}_{j}}\left(W_{e n}\right)_{0}(\mathbf{r}) \delta \mathbf{R}_{j} \\
& -\int d \mathbf{r}\left(W_{e n}\right)_{0}(\mathbf{r}) \delta \rho_{e}(\mathbf{r}),
\end{aligned}
$$

for $k=1, \ldots, N_{n}$. For Maxwell equations, we can write

$$
\begin{aligned}
\left(-\frac{\omega^{2}}{c^{2}}-\nabla^{2}\right) \delta \mathbf{A}(\mathbf{r}, \omega)-\frac{\iota \omega}{c} \nabla \delta \phi(\mathbf{r}, \omega) & =\frac{4 \pi}{c} \delta \mathbf{j}(\mathbf{r}, \omega), \\
-\nabla^{2} \delta \phi(\mathbf{r}, \omega) & =4 \pi \delta \rho(\mathbf{r}, \omega),
\end{aligned}
$$

with

$$
\delta \mathbf{j}=\delta \mathbf{j}_{n}-\delta \mathbf{j}_{e}, \delta \rho=\delta \rho_{n}-\delta \rho_{e} .
$$

The nuclear current and charge densities $\delta \mathbf{j}_{n}$ and $\delta \rho_{n}$ are given respectively as

$$
\begin{gathered}
\delta \mathbf{j}_{n}(\mathbf{R}, \omega)=\sum_{k=1}^{N_{n}} Z_{k}(\iota \omega) \mathrm{I}\left(\mathbf{R}-\mathbf{R}_{0 k}\right) \delta \mathbf{R}_{k}, \\
\delta \rho_{n}(\mathbf{R}, \omega)=-\sum_{k=1}^{N_{n}} Z_{k} \nabla_{\mathbf{R}} \mathrm{I}\left(\mathbf{R}-\mathbf{R}_{0 k}\right) \delta \mathbf{R}_{k} .
\end{gathered}
$$

And the electronic current density $\delta \mathbf{j}_{e}$ and density $\delta \rho_{e}$ are obtained within 
the linear response theory of TD-CDFT $[26,9]$ such that

$$
\begin{aligned}
& \delta \mathbf{j}_{e}(\mathbf{r}, \omega)=\int d \mathbf{r}^{\prime}\left(\chi_{j j}\left(\mathbf{r}, \mathbf{r}^{\prime}, \omega\right)-\chi_{j j}\left(\mathbf{r}, \mathbf{r}^{\prime}, 0\right)\right) \delta \mathbf{A}_{K S}(\mathbf{r}, \omega) \\
&+\int d \mathbf{r}^{\prime} \chi_{j \rho}\left(\mathbf{r}, \mathbf{r}^{\prime}, \omega\right) \delta v_{K S}(\mathbf{r}, \omega) \\
& \delta \rho_{e}(\mathbf{r}, \omega)=\int d \mathbf{r}^{\prime} \chi_{j \rho}\left(\mathbf{r}, \mathbf{r}^{\prime}, \omega\right) \delta \mathbf{A}_{K S}(\mathbf{r}, \omega) \\
&+\int d \mathbf{r}^{\prime} \chi_{\rho \rho}\left(\mathbf{r}, \mathbf{r}^{\prime}, \omega\right) \delta v_{K S}(\mathbf{r}, \omega),
\end{aligned}
$$

where $\chi$ 's are the linear response functions (refer to $[26,9]$ and the Appendix for formulation). $\delta \mathbf{A}_{K S}$ and $\delta v_{K S}$ are functionals of the incident and induced fields with the following form:

$$
\begin{aligned}
\delta \mathbf{A}_{K S}(\mathbf{r}, \omega) & =\mathbf{A}_{0}(\mathbf{r}, \omega)+\delta \mathbf{A}(\mathbf{r}, \omega)+\delta \mathbf{A}_{x c}(\mathbf{r}, \omega) \\
& =\mathbf{A}_{0}(\mathbf{r}, \omega)+\delta \mathbf{A}(\mathbf{r}, \omega)+\int d \mathbf{r}^{\prime} \mathbf{f}_{x c}\left(\mathbf{r}, \mathbf{r}^{\prime}, \omega\right) \delta \mathbf{j}_{e}\left(\mathbf{r}^{\prime}, \omega\right),
\end{aligned}
$$

and

$$
\begin{aligned}
\delta v_{K S}(\mathbf{r}, \omega)= & \delta W_{e n}(\mathbf{r}, \overline{\mathbf{R}})+\delta v_{H}(\mathbf{r}, \omega)+\delta v_{x c}(\mathbf{r}, \omega) \\
= & \delta W_{e n}(\mathbf{r}, \overline{\mathbf{R}})+\int d \mathbf{r}^{\prime} \frac{\delta \rho_{e}\left(\mathbf{r}^{\prime}, \omega\right)}{\left|\mathbf{r}-\mathbf{r}^{\prime}\right|} \\
& +\int d \mathbf{r}^{\prime} f_{x c}\left(\mathbf{r}, \mathbf{r}^{\prime}, \omega\right) \delta \rho_{e}\left(\mathbf{r}^{\prime}, \omega\right),
\end{aligned}
$$

with $\mathbf{f}_{x c}$ and $f_{x c}$ being the vector and scalar exchange-correlation kernels, respectively.

Therefore, the coupled Maxwell-Ehrenfest-Kohn-Sham system within linear response theory consists of equations (31), (32), (33) and (34) for determining the induced EM fields $(\delta \mathbf{A}, \delta \phi)$, nuclear positions $\left\{\delta \mathbf{R}_{k}\right\}$, current and charge densities $\left(\delta \mathbf{j}_{n}, \delta \rho_{n}\right)$ due to nuclei, and current and electron densities $\left(\delta \mathbf{j}_{e}, \delta \rho_{e}\right)$ due to electrons. The linear response formulation helps us to avoid dealing with the time scale separation of the original nonlinear system.

\subsection{P-matrix Formulation and the resonance condition}

To identify the resonant condition for the nano optical-mechanical system, following $[4,2]$, we can formulate the coupled system in a compact form with the $P$-matrix notation and define the resonant frequencies as solutions of an 
eigenvalue problem. We present formulations of $P$-matrix in this section. More details can be found in the Appendix. As in $[4,2]$, we define the $P$-matrix such that

$$
\begin{aligned}
& P_{l m}(\omega)= \\
& \frac{\frac{-\omega}{c\left(\epsilon_{m}-\epsilon_{l}\right)} \int d \mathbf{r} \psi_{l}(\mathbf{r}) \mathbf{j}_{p} \psi_{m}(\mathbf{r}) \delta \mathbf{A}_{K S}(\mathbf{r}, \omega)+\int d \mathbf{r} \psi_{l}(\mathbf{r}) \psi_{m}(\mathbf{r}) \delta v_{K S}(\mathbf{r}, \omega)}{\epsilon_{m}-\epsilon_{l}+\omega},
\end{aligned}
$$

with ground state $\mathrm{KS}$ orbitals $\left\{\psi_{l}, \epsilon_{l}\right\}$ and $\left\{\psi_{m}, \epsilon_{m}\right\}$ and the paramagnetic current density operator $\mathbf{j}_{p}$ is defined as

$$
\mathbf{j}_{p}=-\iota\left(\nabla-\nabla^{\dagger}\right) / 2,
$$

with $\nabla^{\dagger}$ acting on the term to the left. Let $\mathcal{P}_{l m}(\omega) \equiv P_{l m}(\omega)-P_{m l}(\omega)$. As shown in the Appendix, we have

$$
\begin{aligned}
\delta \mathbf{j}_{e}(\mathbf{r}, \omega)= & \sum_{i a} \frac{\omega f_{i}}{\epsilon_{i}-\epsilon_{a}} \psi_{i}(\mathbf{r}) \mathbf{j}_{p} \psi_{a}(\mathbf{r}) \mathcal{P}_{i a}(\omega), \\
\delta \rho_{e}(\mathbf{r}, \omega)= & -\sum_{i a} f_{i} \psi_{i}(\mathbf{r}) \psi_{a}(\mathbf{r}) \mathcal{P}_{i a}(\omega), \\
\delta \mathbf{A}(\mathbf{r}, \omega)= & \int \mathbf{G}\left(\mathbf{r}, \mathbf{r}^{\prime}\right) \delta \mathbf{j}_{n}\left(\mathbf{r}^{\prime}, \omega\right) d \mathbf{r}^{\prime} \\
& -\sum_{i a} \frac{\omega f_{i}}{\left(\epsilon_{i}-\epsilon_{a}\right)} \int \mathbf{G}\left(\mathbf{r}, \mathbf{r}^{\prime}\right) \psi_{i}\left(\mathbf{r}^{\prime}\right) \mathbf{j}_{p} \psi_{a}\left(\mathbf{r}^{\prime}\right) d \mathbf{r}^{\prime} \mathcal{P}_{i a}(\omega), \\
\delta \phi(\mathbf{r}, \omega)= & \int G\left(\mathbf{r}, \mathbf{r}^{\prime}\right) \delta \rho_{n}\left(\mathbf{r}^{\prime}, \omega\right) d \mathbf{r}^{\prime} \\
& +\sum_{i a} f_{i} \int G\left(\mathbf{r}, \mathbf{r}^{\prime}\right) \psi_{i}\left(\mathbf{r}^{\prime}\right) \psi_{a}\left(\mathbf{r}^{\prime}\right) d \mathbf{r}^{\prime} \mathcal{P}_{i a}(\omega) .
\end{aligned}
$$

where $G$ and $\mathbf{G}$ are scalar and tensor Green's functions, respectively, and $i$ and $a$ indicate occupied and unoccupied ground state KS orbitals respectively (hereafter, we use $i, j$ for occupied orbitals and $a, b$ for unoccupied orbitals).

With (39) and (33), from (31) we can write $\delta \mathbf{R}_{k}$ as linear expansions in terms of $\mathcal{P}_{i a}$ such as

$$
\delta \mathbf{R}_{k}=\sum_{i a} \mathbf{C}_{i a}^{\mathbf{R}_{k}} \mathcal{P}_{i a}(\omega)+\mathbf{D}^{\mathbf{R}_{k}}
$$


with $\mathbf{C}^{\mathbf{R}_{k}}$ and $\mathbf{D}^{\mathbf{R}_{k}}$ being appropriate coefficients. Then with (40) and (33), we have

$$
\begin{aligned}
\delta \rho_{n}(\mathbf{R}, \omega)=- & \sum_{i a} \sum_{k=1}^{N_{n}} Z_{k} \nabla_{\mathbf{R}} \mathrm{I}\left(\mathbf{R}-\mathbf{R}_{0 k}\right) \cdot \mathbf{C}_{i a}^{\mathbf{R}_{k}} \mathcal{P}_{i a}(\omega) \\
& -\sum_{k=1}^{N_{n}} Z_{k} \nabla_{\mathbf{R}} \mathrm{I}\left(\mathbf{R}-\mathbf{R}_{0 k}\right) \cdot \mathbf{D}^{\mathbf{R}_{k}}, \\
\delta \mathbf{j}_{n}(\mathbf{R}, \omega)=- & \sum_{i a} \sum_{k=1}^{N_{n}} Z_{k} \iota \omega \mathrm{I}\left(\mathbf{R}-\mathbf{R}_{0 k}\right) \mathbf{C}_{i a}^{\mathbf{R}_{k}} \mathcal{P}_{i a}(\omega) \\
& -\sum_{k=1}^{N_{n}} Z_{k} \iota \omega \mathrm{I}\left(\mathbf{R}-\mathbf{R}_{0 k}\right) \mathbf{D}^{\mathbf{R}_{k}},
\end{aligned}
$$

which can be used to obtain $\delta \mathbf{A}$ and $\delta \phi$ from (39) as

$$
\begin{aligned}
\delta \mathbf{A}(\mathbf{r}, \omega)=\sum_{i a} & \left(-\frac{\omega f_{i}}{\left(\epsilon_{i}-\epsilon_{a}\right)} \int \mathbf{G}\left(\mathbf{r}, \mathbf{r}^{\prime}\right) \psi_{i}\left(\mathbf{r}^{\prime}\right) \mathbf{j}_{p} \psi_{a}\left(\mathbf{r}^{\prime}\right) d \mathbf{r}^{\prime}\right. \\
& \left.-\int \mathbf{G}\left(\mathbf{r}, \mathbf{r}^{\prime}\right)\left(\sum_{k=1}^{N_{n}} Z_{k} \iota \omega \mathrm{I}\left(\mathbf{r}^{\prime}-\mathbf{R}_{0 k}\right) \mathbf{C}_{i a}^{\mathbf{R}_{k}}\right) d \mathbf{r}^{\prime}\right) \mathcal{P}_{i a}(\omega) \\
& -\int \mathbf{G}\left(\mathbf{r}, \mathbf{r}^{\prime}\right) \sum_{k=1}^{N_{n}} Z_{k} \iota \omega \mathrm{I}\left(\mathbf{r}^{\prime}-\mathbf{R}_{0 k}\right) \mathbf{D}^{\mathbf{R}_{k}} d \mathbf{r}^{\prime}, \\
\delta \phi(\mathbf{r}, \omega)= & \sum_{i a}\left(f_{i} \int G\left(\mathbf{r}, \mathbf{r}^{\prime}\right) \psi_{i}\left(\mathbf{r}^{\prime}\right) \psi_{a}\left(\mathbf{r}^{\prime}\right) d \mathbf{r}^{\prime}\right. \\
& \left.-\int G\left(\mathbf{r}, \mathbf{r}^{\prime}\right) \sum_{k=1}^{N_{n}} Z_{k} \nabla_{\mathbf{R}} \mathrm{I}\left(\mathbf{r}^{\prime}-\mathbf{R}_{0 k}\right) \cdot \mathbf{C}_{i a}^{\mathbf{R}_{k}} d \mathbf{r}^{\prime}\right) \mathcal{P}_{i a}(\omega) \\
& -\int G\left(\mathbf{r}, \mathbf{r}^{\prime}\right) \sum_{k=1}^{N_{n}} Z_{k} \nabla_{\mathbf{R}} \mathrm{I}\left(\mathbf{r}^{\prime}-\mathbf{R}_{0 k}\right) \cdot \mathbf{D}^{\mathbf{R}_{k}} d \mathbf{r}^{\prime},
\end{aligned}
$$


and $\delta W_{e n}$ as

$$
\begin{aligned}
\delta W_{e n}(\mathbf{r}, \overline{\mathbf{R}})= & \sum_{k=1}^{N_{n}} \nabla_{\mathbf{R}_{k}} W_{e n}\left(\mathbf{r}, \overline{\mathbf{R}}_{0}\right) \cdot \delta \mathbf{R}_{k} \\
= & \sum_{i a} \sum_{k=1}^{N_{n}} \nabla_{\mathbf{R}_{k}} W_{e n}\left(\mathbf{r}, \overline{\mathbf{R}}_{0}\right) \cdot \mathbf{C}_{i a}^{\mathbf{R}_{k}} \mathcal{P}_{i a}(\omega) \\
& +\sum_{k=1}^{N_{n}} \nabla_{\mathbf{R}_{k}} W_{e n}\left(\mathbf{r}, \overline{\mathbf{R}}_{0}\right) \cdot \mathbf{D}^{\mathbf{R}_{k}}
\end{aligned}
$$

The above quantities $\left\{\delta \mathbf{A}, \delta \phi, \delta \mathbf{j}_{e}, \delta \rho_{e}, \delta \mathbf{j}_{n}, \delta \rho_{n}\right\}$ have similar expressions with $\left\{\mathcal{P}_{i a}\right\}$. For notation's simplicity, we rewrite the above quantities in the following compact forms,

$$
\begin{array}{ll}
\delta \mathbf{A}=\sum_{i a} \mathbf{C}_{i a}^{\mathbf{A}} \mathcal{P}_{i a}+\mathbf{D}^{\mathbf{A}}, & \delta \phi=\sum_{i a} C_{i a}^{\phi} \mathcal{P}_{i a}+D^{\phi}, \\
\delta \mathbf{j}_{e}=\sum_{i a} \mathbf{C}_{i a}^{\mathbf{j}_{e}} \mathcal{P}_{i a}, & \delta \rho_{e}=\sum_{i a} C_{i a}^{\rho_{e}} \mathcal{P}_{i a}, \\
\delta \mathbf{j}_{n}=\sum_{i a} \mathbf{C}_{i a}^{\mathbf{j}_{n}} \mathcal{P}_{i a}+\mathbf{D}^{\mathbf{j}_{n}}, & \delta \rho_{n}=\sum_{i a} C_{i a}^{\rho_{n}} \mathcal{P}_{i a}+D^{\rho_{n}}, \\
\delta W_{e n}=\sum_{i a} C_{i a}^{W_{e n}} \mathcal{P}_{i a}+D^{W_{e n}}, &
\end{array}
$$

where the coefficients $C$ 's and $D$ 's can be derived easily from equations (39), (41), (42), and (43). Then by denoting

$$
\begin{aligned}
& F_{i a}^{\mathbf{A}_{0}}(\omega)=\frac{-\omega}{\epsilon_{a}-\epsilon_{i}} \int \psi_{i}(\mathbf{r}) \mathbf{j}_{p} \psi_{a}(\mathbf{r}) \frac{\mathbf{A}_{0}(\mathbf{r}, \omega)}{c} d \mathbf{r}, \\
& F_{i a}^{\mathbf{A}}(\omega)=\frac{-\omega}{\epsilon_{a}-\epsilon_{i}} \int \psi_{i}(\mathbf{r}) \mathbf{j}_{p} \psi_{a}(\mathbf{r}) \frac{\mathbf{D}^{\mathbf{A}}}{c} d \mathbf{r}, \\
& F_{i a}^{W_{e n}}(\omega)=\int \psi_{i}(\mathbf{r}) \psi_{a}(\mathbf{r}) D^{W_{e n}} d \mathbf{r}^{\prime},
\end{aligned}
$$


and

$$
\begin{aligned}
M_{i a, j b}(\omega)= & \frac{-\omega}{\epsilon_{a}-\epsilon_{i}} \int \psi_{i}(\mathbf{r}) \mathbf{j}_{p} \psi_{a}(\mathbf{r}) \frac{\mathbf{C}_{j b}^{\mathbf{A}}}{c} d \mathbf{r} \\
K_{i a, j b}^{x c}(\omega)= & \frac{-\omega}{\epsilon_{a}-\epsilon_{i}} \iint \psi_{i}(\mathbf{r}) \mathbf{j}_{p} \psi_{a}(\mathbf{r}) \mathbf{f}_{x c}\left(\mathbf{r}, \mathbf{r}^{\prime}, \omega\right) \mathbf{C}_{j b}^{\mathbf{j}_{e}} d \mathbf{r}^{\prime} d \mathbf{r} \\
& \quad+\iint \psi_{i}(\mathbf{r}) \psi_{a}(\mathbf{r}) f_{H x c}\left(\mathbf{r}, \mathbf{r}^{\prime}, \omega\right) C_{j b}^{\rho_{e}} d \mathbf{r}^{\prime} d \mathbf{r}, \\
K_{i a, j b}^{W_{e n}}(\omega)= & \int \psi_{i}(\mathbf{r}) \psi_{a}(\mathbf{r}) C_{j b}^{W_{e n}} d \mathbf{r},
\end{aligned}
$$

with $f_{H x c}\left(\mathbf{r}, \mathbf{r}^{\prime}, \omega\right)=1 /\left|\mathbf{r}-\mathbf{r}^{\prime}\right|+f_{x c}\left(\mathbf{r}, \mathbf{r}^{\prime}, \omega\right)$, and letting

$$
F_{i a} \equiv F_{i a}^{\mathbf{A}_{0}}+F_{i a}^{\mathbf{A}}+F_{i a}^{W_{e n}}, \quad K_{i a, j b} \equiv K_{i a, j b}^{x c}+K_{i a, j b}^{W_{e n}}
$$

we have a linear system on the $P$-matrix $[\mathcal{P}]_{i a}$ (see the Appendix for more details on the derivation),

$$
\left(\mathbf{S}-\omega^{2} \mathbf{I}\right) \mathcal{P}=\mathcal{F}
$$

with

$$
\begin{aligned}
& \mathbf{S}_{i a, j b}=\left(\epsilon_{a}-\epsilon_{i}\right)^{2} \delta_{i j} \delta_{a b}-2\left(\epsilon_{a}-\epsilon_{i}\right)\left(M_{i a, j b}+K_{i a, j b}\right), \\
& \mathcal{F}_{i a}=2\left(\epsilon_{a}-\epsilon_{i}\right) F_{i a},
\end{aligned}
$$

and

$$
\delta_{l m}= \begin{cases}1, & \text { if } l=m \\ 0, & \text { else }\end{cases}
$$

Technically, given any incident light with frequency $\omega$, the system can be solved such that $\{\delta \mathbf{A}, \delta \phi\},\left\{\delta \mathbf{R}_{k}\right\}_{k=1}^{N_{n}},\left\{\delta \mathbf{j}_{n}, \delta \rho_{n}\right\}$, and $\left\{\delta \mathbf{j}_{e}, \delta \rho_{e}\right\}$ are determined. Furthermore, the linear system (48) also enables us to determine the resonant eigenmodes of the light-matter interactions. Resonant eigenmodes exist for particular frequencies such that the matrix in (48) is degenerate, which are called self-sustaining (SS) modes [6]. The resonant structure of optical spectra in general can be determined by the SS modes. Therefore, we can solve

$$
\operatorname{det}\left(\mathcal{S}-\omega^{2} \mathbf{I}\right)=0
$$

to determine the eigenfrequencies $\omega$. In particular, we can treat it as an eigenvalue problem to compute the eigenvalues of the matrix $\mathcal{S}$. 


\section{A Self-consistent Multiscale Method}

In this section, we present a numerical scheme to solve the linear system simplified as (48) for optical-mechanical responses of nanostructures, and the corresponding eigenvalue problem (51) for resonant frequencies. The lightmatter interaction is multiscale in nature with physical processes from well separated time and space scales coupled together. The linearized MaxwellEhrenfest-Kohn-Sham system inherits the multiscale property such that the EM fields extend on the macroscopic domain, while the molecular and electronic structures vary in a microscopic region. As shown in [2], a direct discretization of the system with a uniform mesh will lead to a stiff matrix that is numerically unstable. To determine $(\delta \mathbf{A}, \delta \phi), \delta \overline{\mathbf{R}}$, and $(\delta \mathbf{j}, \delta \rho)$ self-consistently, following the multiscale solver presented in [2], a multiscale scheme that seamlessly bridges different scales has the following procedure at each step $m$ :

1. Micro sovler: Given macroscopic quantities $\left(\delta \phi^{m}, \delta \mathbf{A}^{m}\right)$ as parameters, the micro solver iterates between the electronic and molecular equations such that

- Fix molecular variables $\left(\delta \overline{\mathbf{R}}^{m}, \delta \mathbf{j}_{n}^{m}, \delta \rho_{n}^{m}\right)$, and obtain electronic information $\left(\delta \mathbf{j}_{e}^{m+1}, \delta \rho_{e}^{m+1}\right)$ within the linear response of TD-CDFT (first two equations of (39));

- Use electronic information $\left(\delta \mathbf{j}_{e}^{m}, \delta \rho_{e}^{m}\right)$ to get molecular variables $\left\{\delta \mathbf{R}_{k}^{m+1}\right\}$ from Hamilton's equation (31) and $\left(\delta \mathbf{j}_{n}^{m+1}, \delta \rho_{n}^{m+1}\right)$ from equation (33); the Hamilton's equation (31) is solved iteratively to obtain $\left\{\delta \mathbf{R}_{k}^{m+1}\right\}$;

2. Macro solver: With microscopic inputs $\left(\delta \mathbf{j}^{m+1}, \delta \rho^{m+1}\right)$, compute the macroscopic variables $\left(\delta \phi^{m+1}, \delta \mathbf{A}^{m+1}\right)$ as solutions of Maxwell equations (32);

3. Repeat until self-consistent convergence.

The micro solver is performed on a refined mesh covering a smaller domain enclosing the matter, while the macro solver is performed in a larger domain partitioned with coarse meshes. Linear interpolation is used for the communication between numerical solutions of the micro and macro solvers.

For the micro solver, notice that the linear response of TD-CDFT is performed by solving the linear system of $P$-matrix with all other quantities given as fixed. The matrix $\mathcal{S}$ is too complicated to be implemented explicitly 
by directly evaluating the entries which requires intense computational complexity, but the matrix-vector product $\mathcal{S} \cdot \mathcal{P}$ can be obtained for any vector $\mathcal{P}$ as the following:

$$
\begin{aligned}
\sum_{j b} K_{i a, j b}^{x c} \mathcal{P}_{j p}= & \frac{-\omega}{c\left(\epsilon_{a}-\epsilon_{i}\right)} \int \psi_{i}(\mathbf{r}) \mathbf{j}_{p} \psi_{a}(\mathbf{r}) \delta \mathbf{A}_{x c}(\mathbf{r}) d \mathbf{r} \\
& +\int \psi_{i} \mathbf{r} \psi_{a}(\mathbf{r})\left(\delta v_{H}(\mathbf{r})+\delta v_{x c}(\mathbf{r})\right) d \mathbf{r} \\
\sum_{j b} K_{i a, j b}^{W_{e n}} \mathcal{P}_{j b}= & \int \psi_{i}(\mathbf{r}) \psi_{a}(\mathbf{r}) \delta W_{e n} d \mathbf{r}-F_{i a}^{W_{e n}} \\
\sum_{j b} M_{i a, j b} \mathcal{P}_{j b}= & \frac{-\omega}{c\left(\epsilon_{a}-\epsilon_{i}\right)} \int \psi_{i}(\mathbf{r}) \mathbf{j}_{p} \psi_{a}(\mathbf{r}) \delta \mathbf{A}(\mathbf{r}) d \mathbf{r}-F_{i a}^{\mathbf{A}}
\end{aligned}
$$

Therefore, Krylov subspace methods such as the BiCGSTAB algorithm [27] can be used to solve the linear system. The update of $\delta \overline{\mathbf{R}}$ is simply obtained by inverting (31). For the macro solver, Maxwell equations (32) are solved by a hybrid nodal-vector finite element method with the impedance boundary condition imposed [15], i.e.,

$$
\mathbf{n} \times(\nabla \times \mathbf{E})-\iota \frac{\omega}{c} \mathbf{n} \times(\mathbf{n} \times \mathbf{E})=0,
$$

where $\mathbf{n}$ is the outward normal to the boundary, and the EM fields $\mathbf{E}$ and $\mathbf{B}$, are given in the frequency domain by

$$
\mathbf{E}(\mathbf{r})=-\nabla \phi(\mathbf{r})+\frac{\iota \omega \mathbf{A}}{c} \text {, and } \mathbf{B}(\mathbf{r})=\nabla \times \mathbf{A}(\mathbf{r}) .
$$

For determining the resonant conditions, we adopt the same self-consistent strategy for the nonlinear eigenvalue problem (51) as in [2]. Starting with an initial guess of the frequency (eigenvalue) $\omega_{0}$, fixing $\mathcal{S}\left(\omega_{0}\right)$, a linear eigenvalue problem is solved with a Jacobi-Davidson algorithm [1] to update $\mathcal{S}$. The scheme is iterated until convergence.

In this work, we assume the system is in vacuum. At the microscopic level, the infinite space domain is truncated with a sphere containing the molecular structures under consideration. We use the package OCTOPUS [5], which adopts real-space finite-difference methods on structured meshes, to obtain the ground state KS orbitals by solving the ground state KS equations (19). 
Periodic boundary conditions are imposed in OCTOPUS for our applications. For the macroscopic variables, Maxwell equations are solved with a hybrid nodal-vector finite element method on a triangulated mesh (e.g., see [15]). Figure 1 demonstrates the domains and meshes for macro and micro solvers. It can be seen that a larger domain with coarse meshes is used for macro solver, while a smaller domain with refined meshes is used for micro solver.

(a)

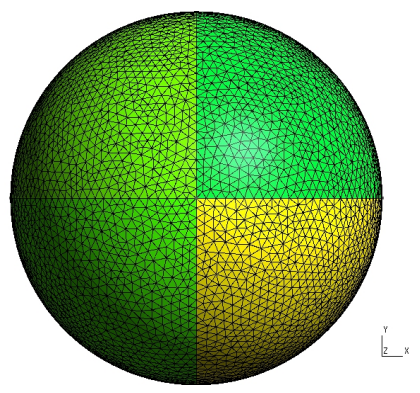

(b)

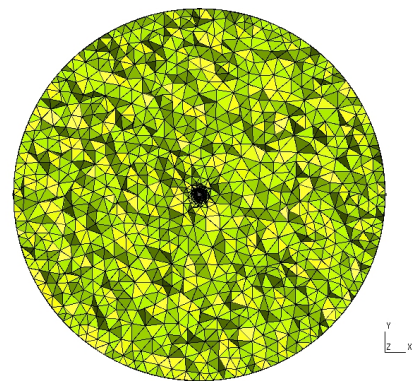

(c)

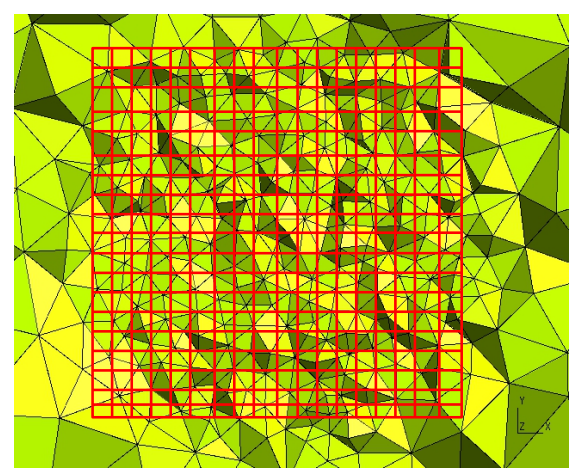

Figure 1: Domain and meshes. (a): spherical domain and triangulated mesh for Maxwell equations, the domain is centered at the nanostructure; (b): a slice of the mesh at $z=0$, the mesh is refined at the middle where the nanostructure is located; and (c): zoom-in of the center and mesh, and rectangular mesh for TD-CDFT.

\section{Numerical Examples}

We illustrate the Maxwell-Ehrenfest-Kohn-Sham model with the nanostructure chosen as trans-Azobenzene as shown in Figure 2 with $x-y$ - $z$ coordinates listed in Appendix B. The multiscale scheme is used to solve the linear response formulation (48). For solving the linear system, BiCGSTAB algorithm is incorporated into the multiscale scheme; for solving the eigenvalue 


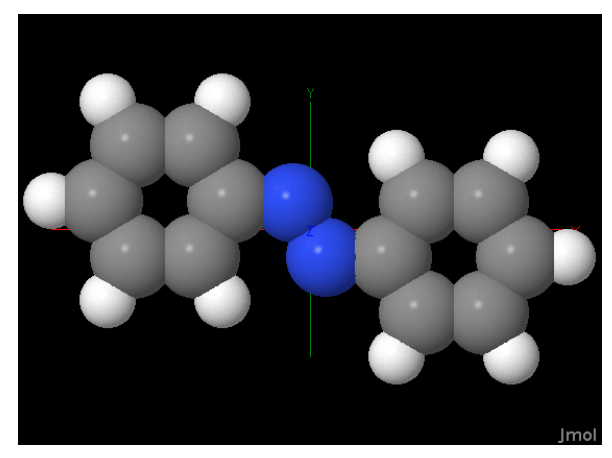

Figure 2: The sample trans-Azobenzene $\left(C_{12} H_{10} N_{2}\right)$ is shown on $x y$ plane. The sample is centered at $(0,0,0)$.

problem, the Jacobi-Davidson algorithm is incorporated into the multiscale scheme. The spherical domain for Maxwell equations is centered at $(0,0,0)$ with radius 40nm, and discretized by a triangulated mesh with 21971 nodes and 127412 tetrahedrons. The cubic domain for TD-CDFT is $[-15,15]^{3}$ atomic units (a.u.), discretized by a uniform mesh of mesh size 0.5 a.u. for ground state in OCTOPUS, and 1 a.u. for linear responses. Figure 1 illustrates numerical domains and meshes.

The lowest eigenfrequency is computed by the Jacobi-Davidson algorithm and is approximately 0.1425 a.u. $(\approx 3.8776 \mathrm{ev}, 318 \mathrm{~nm})$, which is in the wavelength of UV light and is consistent with experiments [30]. Figure 3 shows the linear response spectra computed by solving the linear system with given incident light with different frequencies $\omega$, incident directions $\mathbf{d}$ and polarizations $\mathbf{p}$ such that

$$
\mathbf{A}_{0}(\mathbf{r}, \omega)=\frac{c}{\iota \omega} \mathbf{p} e^{\iota \frac{\omega}{c} \mathbf{r} \cdot \mathbf{d}}
$$

From Figure 3, we observe peaks at the frequency that is approximately the eigenfrequency computed with the Jacobi-Davidson algorithm. When the frequency approaches the eigenfrequency, the nuclei intend to move. This is consistent with the azobenzene photoisomerization, i.e., the trans form can be converted to the cis form using an appropriate wavelength (UV at 300400nm) of light [13]. The magnitude depends on the incident light. Also, the magnitude of the dipole moment depends on the incident light. Different 
configurations for the incident light are tested and the result seems to be robust.

(a)
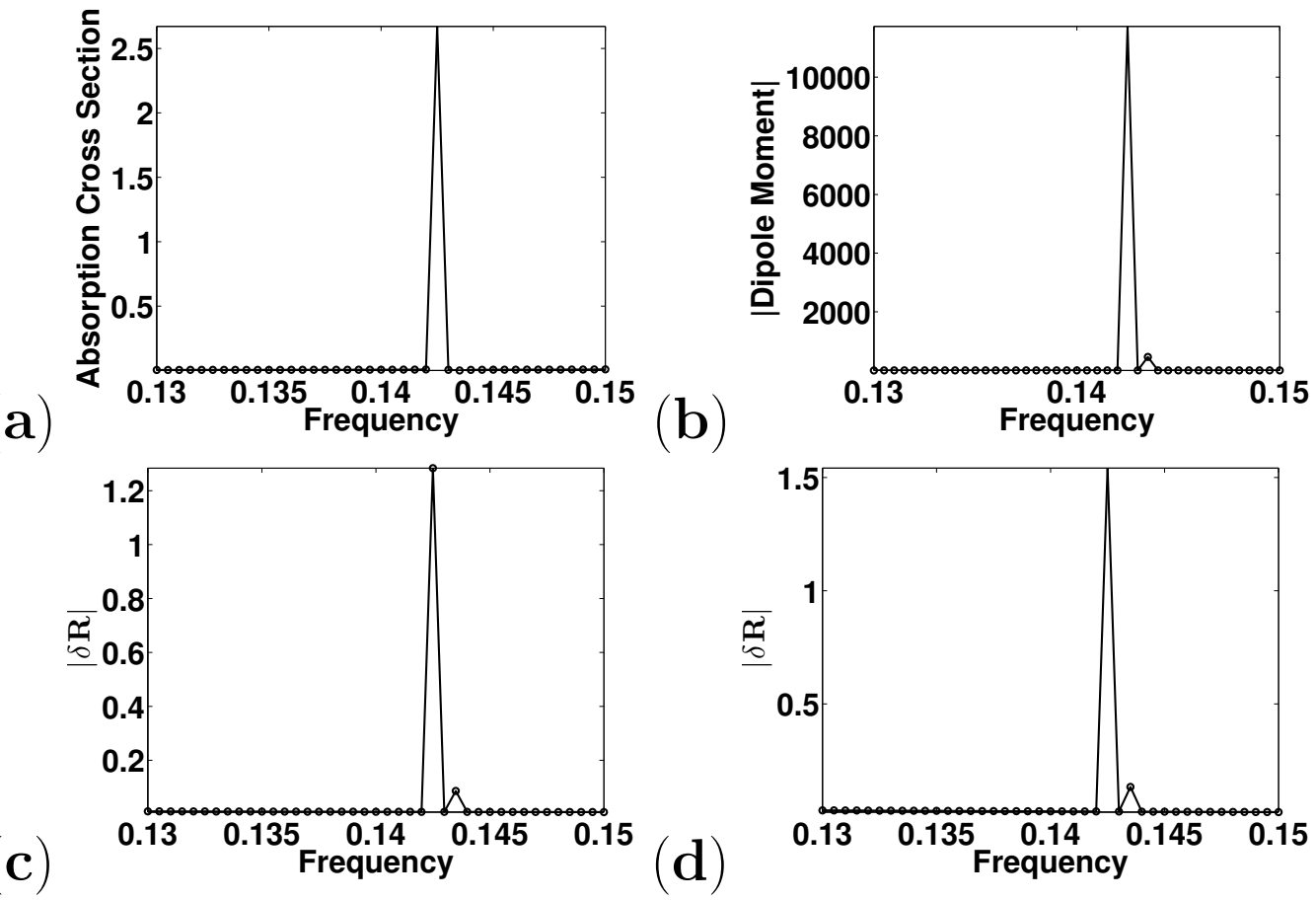

Figure 3: Linear response spectra. (a) shows the absorption cross section. Incident light configuration is $\mathbf{d}=(0,1,0), \mathbf{p}=(1,0,0)$; (b) gives the magnitude of dipole moments; (c)-(d) illustrate the magnitude of $\delta \mathbf{R}_{k}$ for Nitrogen 1 and Nitrogen 2, respectively.

\section{Conclusion}

A numerically tractable semi-classical model is introduced for studying optical responses of nanostructures such that the EM field is described classically and the matter is treated quantum mechanically. The Ehrenfest molecular dynamics and Time-Dependent Current Density Functional Theory are adopted to avoid the difficulty of solving high dimensional Schrödinger equation. The so obtained Maxwell-Ehrenfest-Kohn-Sham system is further linearized, which facilitates the computation of resonant frequencies. To deal with the multiscale nature of the system, a multiscale scheme is introduced and numerical examples are presented. Future work will involve plasmonic metal enhancement and extended systems. 


\section{References}

[1] Z. Bai, J. Demmel, J. Dongarra, A. Ruhe, H. van der Vorst (editors), Templates for the Solution of Eigenvalue Problems: A Practical Guide, SIAM, 2000.

[2] G. Bao, D. Liu, S. Luo, A Multiscale Method for Optical Responses of Nanostructures, SIAM J. Appl. Math. 72 (2013) 741-756.

[3] G.F. Bertsch, J.I. Iwata, A. Rubio, K. Yabana, Real-space, real-time method for the dielectric function, Physical Review B 62 (2000) 79988002.

[4] M.E. Casida, Time-Dependent Density Functional Response Theory for Molecules, In Recent advances in density functional methods; D. P. Chong, Ed. World Scientific, Singapore (1995) 155-193.

[5] A. Castro, H. Appel, M. Oliveira, C.A. Rozzi, X. Andrade, F. Lorenzen, M.A.L. Marques, octopus: a tool for the application of time-dependent density functional theory, Phys. Stat. Sol. B 243 (2006) 2465-2488.

[6] K. Cho, Optical Response of Nanostructures: Microscopic Nonlocal Theory, Springer, New York, 2003.

[7] C. Cohen-Tannoudji, J. Dupont-Roc, G. Grynberg, Photons and Atoms: Introduction to Quantum Electrodynamics, Wiley, New York, 1989.

[8] W. E, J. Lu, X. Yang, Effective Maxwell equations from time-dependent density functional theory, Acta Mathematica Sinica 27 (2011) 339-368.

[9] M. van Faassen, P.L. de Boeij, R. van Leeuwen, J.A. Berger, J.G. Snijders, Ultranonlocality in Time-Dependent Current-Density-Functional Theory: Application to Conjugated Polymers, Phys. Rev. Lett. 88 (2002) 186401-186404.

[10] C. Fiolhais, F. Nogueira, M.A.L. Marques(editors), A Primer in Density functional Theory, Lect. Notes Phys. 620, Springer-Verlag, New York, 2003.

[11] S.K. Ghosh, A.K. Dhara, Density-functional theory of many-electron systems subjected to time-dependent electric and magnetic fields, Phys. Rev. A 38 (1988) 1149-1158. 
[12] P. Gorostiza, E. Isacoff, Optical switches for remote and noninvasive control of cell signaling, Science 322 (2008) 395-399.

[13] G.S. Hartley, The cis form of Azobenzene, Nature 140 (1937) 281.

[14] P. Hohenberg, W. Kohn, Inhomogeneous Electron Gas, Phys. Rev. 136 (1964) 864-871.

[15] J.M. Jin, The Finite Element Method in Electromagnetics, (2nd Ed.), New York: John Wiley \& Sons, 2002.

[16] H. Kang, H. Liu, J.A. Phillips, Z. Cao, Y. Kim, Y. Chen, Z. Yang, J. Li, W. Tan, Single-DNA molecule nanomotor regulated by photons, Nano lett. 9 (2009) 2690-2696.

[17] O. Keller, Local fields in the electrodynamics of mesoscopic media, Phys. Rep. 268 (1996) 85-262.

[18] W. Kohn, L.J. Sham, Self-Consistent Equations Including Exchange and Correlation Effects, Phys. Rev. 140 (1965) 1133-1138.

[19] N.T. Maitra, I. Souza, K. Burke, Current-density functional theory of the response of solids, Physical Review B 68 (2003) 045109.

[20] M.A.L. Marques, C.A. Ullrich, F. Nogueira, A. Rubio, K. Burke, E.K.U.G. (editors), Time-Dependent Density Functional Theory, Lect. Notes. Phys. 706, Springer, Heidelberg, 2006.

[21] D. Marx, J. Hutter, Ab initio molecular dynamics, Cambridge University Press, Cambridge, 2009.

[22] E. Runge, E.K.U. Gross, Density-Functional Theory for TimeDependent Systems, Phys. Rev. Lett. 52 (1984) 997-1000.

[23] A. Stahl, I. Balslev, Electrodynamics of the Semiconductor Band Edge, Springer Tract in Mod. Phys. 110, Springer-Verlag, New York, 1987.

[24] C.A. Ullrich, Time-Dependent Density-Functional Theory: Concepts and Applications, Oxford University Press, Oxford, New York, 2011.

[25] G. Vignale, Current-Dependent Exchange-Correlation Potential for Dynamical Linear Response Theory, Phys. Rev. Lett. 77 (1996) 2037-2040. 
[26] G. Vignale, C.A. Ullrich, S. Conti, Time-Dependent Density Functional Theory Beyond the Adiabatic Local Density Approximation, Phys. Rev. Lett. 79 (1997) 4878-4881.

[27] H.A.V.D. Vorst, Bi-CGSTAB: a fast and smoothly converging variant of Bi-CG for the solution of nonsymmetric linear systems, SIAM J. Sci. Stat. Comput. 13 (1992) 631-644.

[28] S.H. Vosko, L. Wilk, M. Nusair, Accurate spin-dependent electron liquid correlation energies for local spin density calculations: a critical analysis, Can. J. Phys. 58 (1980) 1200-1211.

[29] T. Wu, T.A. Nieminen, S. Mohanty, J. Miotke, R.L. Meyer, H. Rubinsztein-Dunlop, M.W. Berns, A photon-driven micro motor can direct nerve fibre growth, Nature Photonics 6 (2012) 62-67.

[30] Q. Yuan, Y. Zhang, Y. Chen, R. Wang, C. Du, E. Yasun, W. Tan, Using silver nanowire antennas to enhance then conversion efficiency of photo responsive DNA nanomotors, Proc. Natl. Acad. Sci. 108 (2011) 9331-9336.

\section{Appendix A. Formulations with $\boldsymbol{P}$-matrix}

The linear response function $\chi$ in (34) is given by

$$
\begin{aligned}
\chi_{\alpha \beta}\left(\mathbf{r}, \mathbf{r}^{\prime}, \omega\right)= & \sum_{i a} f_{i}\left[\left(\frac{\psi_{i}(\mathbf{r}) \alpha \psi_{a}(\mathbf{r}) \psi_{a}\left(\mathbf{r}^{\prime}\right) \beta \psi_{i}\left(\mathbf{r}^{\prime}\right)}{\epsilon_{i}-\epsilon_{a}+\omega}\right)\right. \\
& \left.-\left(\frac{\psi_{i}(\mathbf{r}) \alpha \psi_{a}(\mathbf{r}) \psi_{a}\left(\mathbf{r}^{\prime}\right) \beta \psi_{i}\left(\mathbf{r}^{\prime}\right)}{\epsilon_{a}-\epsilon_{i}+\omega}\right)^{*}\right],
\end{aligned}
$$

where $i$ and $a$ run over the occupied and unoccupied ground state KS orbitals of (19), respectively, and $\alpha$ and $\beta$ are either the electron density operator 
$\boldsymbol{\rho}=1$ or the paramagnetic current density operator $\mathbf{j}_{p}$. So we have

$$
\begin{aligned}
\delta \rho(\mathbf{r}, \omega)= & \int \sum_{i a} f_{i}\left[\left(\frac{\psi_{i}(\mathbf{r}) \psi_{a}(\mathbf{r}) \psi_{a}\left(\mathbf{r}^{\prime}\right) \mathbf{j}_{p} \psi_{i}\left(\mathbf{r}^{\prime}\right)}{\epsilon_{i}-\epsilon_{a}+\omega}\right)\right. \\
& \left.-\left(\frac{\psi_{i}(\mathbf{r}) \psi_{a}(\mathbf{r}) \psi_{a}\left(\mathbf{r}^{\prime}\right) \mathbf{j}_{p} \psi_{i}\left(\mathbf{r}^{\prime}\right)}{\epsilon_{a}-\epsilon_{i}+\omega}\right)^{*}\right] \cdot \delta \mathbf{A}_{K S}\left(\mathbf{r}^{\prime}, \omega\right) d \mathbf{r}^{\prime} \\
& +\int \sum_{i a} f_{i}\left[\left(\frac{\psi_{i}(\mathbf{r}) \psi_{a}(\mathbf{r}) \psi_{a}\left(\mathbf{r}^{\prime}\right) \psi_{i}\left(\mathbf{r}^{\prime}\right)}{\epsilon_{i}-\epsilon_{a}+\omega}\right)\right. \\
& \left.-\left(\frac{\psi_{i}(\mathbf{r}) \psi_{a}(\mathbf{r}) \psi_{a}\left(\mathbf{r}^{\prime}\right) \psi_{i}\left(\mathbf{r}^{\prime}\right)}{\epsilon_{a}-\epsilon_{i}+\omega}\right)^{*}\right] \delta v_{K S}\left(\mathbf{r}^{\prime}, \omega\right) d \mathbf{r}^{\prime}
\end{aligned}
$$

which is simplified as

$$
\begin{aligned}
& \delta \rho(\mathbf{r}, \omega)=\sum_{i a} f_{i} \psi_{i}(\mathbf{r}) \psi_{a}(\mathbf{r})\left(\frac{1}{\epsilon_{i}-\epsilon_{a}+\omega}\right) \\
& {\left[\frac{-\omega}{c\left(\epsilon_{i}-\epsilon_{a}\right)} \int \psi_{a}\left(\mathbf{r}^{\prime}\right) \mathbf{j}_{p} \psi_{i}\left(\mathbf{r}^{\prime}\right) \cdot \delta \mathbf{A}_{K S}\left(\mathbf{r}^{\prime}, \omega\right) d \mathbf{r}^{\prime}+\int \psi_{a}\left(\mathbf{r}^{\prime}\right) \psi_{i}\left(\mathbf{r}^{\prime}\right) \delta v_{K S}(\mathbf{r}, \omega) d \mathbf{r}\right]} \\
& -f_{i} \psi_{i}(\mathbf{r}) \psi_{a}(\mathbf{r})\left(\frac{1}{\epsilon_{a}-\epsilon_{i}+\omega}\right) \\
& {\left[\frac{-\omega}{c\left(\epsilon_{a}-\epsilon_{i}\right)} \int \psi_{i}\left(\mathbf{r}^{\prime}\right) \mathbf{j}_{p} \psi_{a}\left(\mathbf{r}^{\prime}\right) \cdot \delta \mathbf{A}_{K S}\left(\mathbf{r}^{\prime}, \omega\right) d \mathbf{r}^{\prime}+\int \psi_{a}\left(\mathbf{r}^{\prime}\right) \psi_{i}\left(\mathbf{r}^{\prime}\right) \delta v_{K S}\left(\mathbf{r}^{\prime}, \omega\right) d \mathbf{r}\right] .}
\end{aligned}
$$

Then by definition of $P$-matrix in (37),

$$
\begin{aligned}
\delta \rho(\mathbf{r}, \omega) & =\sum_{i a} f_{i} \psi_{i}(\mathbf{r}) \psi_{a}(\mathbf{r}) P_{a i}-f_{i} \psi_{a}(\mathbf{r}) \psi_{i}(\mathbf{r}) P_{i a} \\
& =\sum_{i a} f_{i} \psi_{i}(\mathbf{r}) \psi_{a}(\mathbf{r})\left[P_{a i}-P_{i a}\right],
\end{aligned}
$$

which is the second equation in (39). The first equation in (39) can be derived similarly.

By presenting the solutions of Maxwell equations (32) as integrals with 
appropriate tensorial Green's function G and scalar Green's function G, i.e.,

$$
\begin{aligned}
\delta \mathbf{A}(\mathbf{r}, \omega) & =\int \mathbf{G}\left(\mathbf{r}, \mathbf{r}^{\prime}\right) \delta \mathbf{j}\left(\mathbf{r}^{\prime}, \omega\right) d \mathbf{r}^{\prime} \\
\delta \phi(\mathbf{r}, \omega) & =\int G\left(\mathbf{r}, \mathbf{r}^{\prime}\right) \delta \rho\left(\mathbf{r}^{\prime}, \omega\right) d \mathbf{r}^{\prime}
\end{aligned}
$$

and using the first two equations of (39) for $\delta \rho_{e}$ and $\delta \mathbf{j}_{e}$, we have the last two equations in (39). From the Hamilton's equation (31), with (33) and (39), we can have a linear system for $\delta \mathbf{R}_{k}$, whose solution can be represented as (40) with $\mathbf{C}^{\mathbf{R}_{k}}$ and $\mathbf{D}^{\mathbf{R}_{k}}$ being appropriate coefficients. With equation (40), we have equation (41), and then equations (42) and (43). By presenting perturbations in compact forms in (44) with appropriate coefficients C's and D's, we can derive a linear system for the $P$-matrix as in $[4,9,2]$. By substituting equation (35)-(36) into equation (37) and using equation (44), we have

$$
\begin{aligned}
& \left(\epsilon_{a}-\epsilon_{i}+\omega\right) P_{i a}= \\
& \frac{-\omega}{\epsilon_{a}-\epsilon_{i}} \int \psi_{i}(\mathbf{r}) \mathbf{j}_{p} \psi_{a}(\mathbf{r}) \frac{\mathbf{A}_{0}(\mathbf{r}, \omega)}{c} d \mathbf{r}+\frac{-\omega}{\epsilon_{a}-\epsilon_{i}} \int \psi_{i}(\mathbf{r}) \mathbf{j}_{p} \psi_{a}(\mathbf{r}) \frac{\mathbf{D}^{\mathbf{A}}}{c} d \mathbf{r} \\
& +\int \psi_{i}(\mathbf{r}) \psi_{a}(\mathbf{r}) D^{W_{e n}} d \mathbf{r}+\sum_{j b} \frac{-\omega}{\epsilon_{a}-\epsilon_{i}} \int \psi_{i}(\mathbf{r}) \mathbf{j}_{p} \psi_{a}(\mathbf{r}) \frac{\mathbf{C}_{j b}^{\mathbf{A}}}{c} d \mathbf{r} \mathcal{P}_{j b} \\
& +\sum_{j b} \frac{-\omega}{\epsilon_{a}-\epsilon_{i}} \iint \psi_{i}(\mathbf{r}) \mathbf{j}_{p} \psi_{a}(\mathbf{r}) \mathbf{f}_{x c}\left(\mathbf{r}, \mathbf{r}^{\prime}, \omega\right) \mathbf{C}_{j b}^{\mathbf{j}_{e}} d \mathbf{r}^{\prime} d \mathbf{r} \mathcal{P}_{j b} \\
& +\sum_{j b} \int \psi_{i}(\mathbf{r}) \psi_{a}(\mathbf{r}) C_{j b}^{W_{e n}} d \mathbf{r} \mathcal{P}_{j b} \\
& +\sum_{j b} \iint \psi_{i}(\mathbf{r}) \psi_{a}(\mathbf{r}) f_{H x c}\left(\mathbf{r}, \mathbf{r}^{\prime}, \omega\right) C_{j b}^{\rho_{e}} d \mathbf{r}^{\prime} d \mathbf{r} \mathcal{P}_{j b} .
\end{aligned}
$$


A similar equation can be derived for $P_{a i}$ as

$$
\begin{aligned}
& \left(\epsilon_{i}-\epsilon_{a}+\omega\right) P_{a i}= \\
& \frac{-\omega}{\epsilon_{i}-\epsilon_{a}} \int \psi_{a}(\mathbf{r}) \mathbf{j}_{p} \psi_{i}(\mathbf{r}) \frac{\mathbf{A}_{0}(\mathbf{r}, \omega)}{c} d \mathbf{r}+\frac{-\omega}{\epsilon_{i}-\epsilon_{a}} \int \psi_{a}(\mathbf{r}) \mathbf{j}_{p} \psi_{i}(\mathbf{r}) \frac{\mathbf{D}^{\mathbf{A}}}{c} d \mathbf{r} \\
& +\int \psi_{a}(\mathbf{r}) \psi_{i}(\mathbf{r}) D^{W_{e n}} d \mathbf{r}+\sum_{j b} \frac{-\omega}{\epsilon_{i}-\epsilon_{a}} \int \psi_{a}(\mathbf{r}) \mathbf{j}_{p} \psi_{i}(\mathbf{r}) \frac{\mathbf{C}_{j b}^{\mathbf{A}}}{c} d \mathbf{r} \mathcal{P}_{j b} \\
& +\sum_{j b} \frac{-\omega}{\epsilon_{i}-\epsilon_{a}} \iint \psi_{a}(\mathbf{r}) \mathbf{j}_{p} \psi_{i}(\mathbf{r}) \mathbf{f}_{x c}\left(\mathbf{r}, \mathbf{r}^{\prime}, \omega\right) \mathbf{C}_{j b}^{\mathbf{j}_{e}} d \mathbf{r}^{\prime} d \mathbf{r} \mathcal{P}_{j b} \\
& +\sum_{j b} \int \psi_{a}(\mathbf{r}) \psi_{i}(\mathbf{r}) C_{j b}^{W_{e n}} d \mathbf{r} \mathcal{P}_{j b} \\
& +\sum_{j b} \iint \psi_{a}(\mathbf{r}) \psi_{i}(\mathbf{r}) f_{H x c}\left(\mathbf{r}, \mathbf{r}^{\prime}, \omega\right) C_{j b}^{\rho_{e}} d \mathbf{r}^{\prime} d \mathbf{r} \mathcal{P}_{j b} .
\end{aligned}
$$

With notations and definitions in (45)-(45), (A.2) and (A.3) can be written as

$$
\begin{aligned}
& \left(\epsilon_{i}-\epsilon_{a}+\omega\right) P_{a i}=F_{i a}+\sum_{j b}\left(M_{i a, j b}+K_{i a, j b}\right) \mathcal{P}_{j b} \\
& \left(\epsilon_{a}-\epsilon_{i}+\omega\right) P_{i a}=F_{i a}+\sum_{j b}\left(M_{i a, j b}+K_{i a, j b}\right) \mathcal{P}_{j b}
\end{aligned}
$$

from which by subtraction and addition, we obtain a linear system for $\left\{\mathcal{P}_{i a}\right\}$,

$$
\begin{aligned}
& \frac{-\omega^{2}}{\epsilon_{a}-\epsilon_{i}} \mathcal{P}_{i a}+\left(\epsilon_{a}-\epsilon_{i}\right) \mathcal{P}_{i a}-2 \sum_{j b}\left(M_{i a, j b}+K_{i a, j b}\right) \mathcal{P}_{j b}=2 F_{i a} \\
& \sum_{j b}\left\{\left[\left(\epsilon_{a}-\epsilon_{i}\right)^{2} \delta_{i j} \delta_{a b}-2\left(\epsilon_{a}-\epsilon_{i}\right)\left(M_{i a, j b}+K_{i a, j b}\right)\right]-\omega^{2}\right\} \mathcal{P}_{j b}=2\left(\epsilon_{a}-\epsilon_{i}\right) F_{i a} .
\end{aligned}
$$

In a compact form, we have equation (48).

\section{Appendix B. Cartesian Coordinates of the Nanostructure}

The parameters of trans-Azobenzene in the numerical experiment are listed in Table B.1, which is adopted from The National Institute of Stan-

dards and Technology (NIST) Chemistry WebBook (http://webbook.nist.gov/chemistry/). 


\begin{tabular}{ccccc}
\hline Atom Number & Atom & $x$-coordinate & $y$-coordinate & $z$-coordinate \\
\hline 1 & $\mathrm{C}$ & 8.1028 & -1.0595 & 0 \\
2 & $\mathrm{C}$ & 6.8562 & 1.0994 & 0 \\
3 & $\mathrm{C}$ & 4.3631 & 1.0994 & 0 \\
4 & $\mathrm{C}$ & 3.1166 & -1.0595 & 0 \\
5 & $\mathrm{C}$ & 4.3631 & -3.2185 & 0 \\
6 & $\mathrm{C}$ & 6.8562 & -3.2185 & 0 \\
7 & $\mathrm{H}$ & 10.0365 & -1.0595 & 0 \\
8 & $\mathrm{H}$ & 7.8245 & 2.7748 & 0 \\
9 & $\mathrm{H}$ & 3.3948 & 2.7748 & 0 \\
10 & $\mathrm{H}$ & 3.3948 & -4.8938 & 0 \\
11 & $\mathrm{H}$ & 7.8245 & -4.8938 & 0 \\
12 & $\mathrm{C}$ & -3.1161 & 1.1220 & 0 \\
13 & $\mathrm{C}$ & -4.3627 & 3.2810 & 0 \\
14 & $\mathrm{C}$ & -6.8559 & 3.2810 & 0 \\
15 & $\mathrm{C}$ & -8.1025 & 1.1220 & 0 \\
16 & $\mathrm{C}$ & -6.8559 & -1.0371 & 0 \\
17 & $\mathrm{C}$ & -4.3627 & -1.0371 & 0 \\
18 & $\mathrm{H}$ & -3.3943 & 4.9564 & 0 \\
19 & $\mathrm{H}$ & -7.8242 & 4.9564 & 0 \\
20 & $\mathrm{H}$ & -10.0363 & 1.1220 & 0 \\
21 & $\mathrm{H}$ & -7.8242 & -2.7125 & 0 \\
22 & $\mathrm{H}$ & -3.3943 & -2.7125 & 0 \\
23 & $\mathrm{~N}$ & -0.6232 & 1.0597 & 0 \\
24 & $\mathrm{~N}$ & 0.6232 & -1.0597 & 0 \\
\hline & & & & \\
\hline
\end{tabular}

Table B.1: Cartesian coordinates (a.u. units) for trans-Azobenzene. 
LaTeX Source Files for Revision
Click here to download LaTeX Source Files: FilesInRevision.zip

LaTeX Source Files for Revision
Click here to download LaTeX Source Files: FilesInRevision.zip

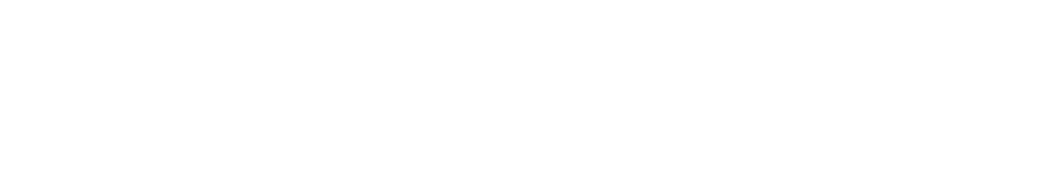

